\title{
CITOLOGÍA DE LA CERVICITIS FOLICULAR Diagnósticos diferenciales
}

\author{
Maritza Johana Arcos Becerra, Nancy Rosario Cerón Camacho*
}

\section{Resumen}

Uno de los problemas más frecuentes que se presentan al realizar la lectura de un extendido citológico son los diagnósticos diferenciales. Esta investigación tiene como objetivo describir los hallazgos y cambios citológicos encontrados en la cervicitis folicular y el diagnóstico diferencial. Las muestras se obtuvieron en un laboratorio citopatológico particular de Bogotá, a las cuales se les realizaron las correspondientes lecturas teniendo en cuenta las siguientes variables: característica celular, descamación celular, citoplasma, núcleo, fondo y elementos celulares no epiteliales. Los resultados fueron: el núcleo del $94 \%$ de los linfocitos en la cervicitis folicular se observó pequeño y grande en el 6\%. La cromatina fue granular en el 70\%, homogénea $28 \%$ e hipercromática en $2 \%$. Los elementos celulares no epiteliales estuvieron representados por plasmocitos $12 \%$, histiocitos $20 \%$ y macrófagos con cuerpos tingibles en un $68 \%$. Los cambios morfológicos de las células escamosas se asociaron con procesos reactivos de inflamación. Los resultados de los diagnósticos diferenciales se analizaron en forma descriptiva teniendo en cuenta la característica celular (borde mal definido), la descamación celular (conglomerados y sincitios), el citoplasma escaso y en algunas ocasiones vacuolado, el núcleo pequeño, redondo y oval, y el fondo en las lesiones malignas con diátesis tumoral, y en los procesos reactivos limpio y en algunos casos inflamatorio.

\section{Introducción}

El interés para realizar esta investigación se debe a que la cervicitis folicular presenta problemas de diagnóstico diferencial tanto con lesiones malignas como el carcinoma no queratinizante de célula pequeña, linfomas, leucemias y adenocarcinoma de endometrio, y con procesos benignos como la hiperplasia de células de reserva, metaplasia escamosa inmadura, atrófia senil, células del estroma endometrial, éxodo endometrial y células endocervicales degeneradas (Figuras 1 a 5).

La cervicitis folicular también denominada linfocitaria es una entidad que se caracteriza por la presencia de abundantes folículos linfoides en el estroma subyacente al epitelio. Una de las formas para evidenciar los folículos bien constituidos son las biopsias cervicales, pero para que en las muestras citológicas aparezcan las células linfoides del folículo debe haber alguna ulceración de la mucosa, la que permitirá que estos elementos celulares se descamen, ya sea en forma aislada o en conglomerados.

* Estudiantes de último semestre de Citohistotecnología.

* Este trabajo fue realizado bajo la tutoría del Dr. Alfredo Romero, Médico Patólogo del Instituto Nacional de Cancerología. Profesor Asistente Fundación Universitaria de Ciencias de la Salud, y de la Sra. Myriam Puerto de Amaya, Bacterióloga, Secretaria Académica y Profesora Asistente de la Fundación Universitaria de Ciencias de la Salud.
Las muestras cervicovaginales en esta entidad se caracterizan por la presencia de un extendido inflamatorio polimorfo, poco cohesivo, que contiene linfocitos grandes y pequeños, células plasmáticas e histiocitos. También es posible observar leucocitos polimorfonucleares neutrófilos, algunas células en mitosis y macrófagos, estos últimos con abundante citoplasma en el que se aprecia fagocitosis, conocidos con el nombre de macrófagos con cuerpos tingibles.

La afección se presenta en mujeres con frotis atróficos, en aquellas que tienen infección por Chlamydia Trachomatis, en portadoras de dispositivo intrauterino (DIU) o aquellas que han sufrido trauma cervical debido al uso de diafragmas.

\section{Materiales y métodos}

Lainvestigación se realizó en 50 muestras de cervicitis folicular y 24 corespondientes a los diagnósticos diferenciales enunciados. Las muestras las proporcionó un laboratorio citopatológico particular de Bogotá entre octubre de 2002 y mayo de 2003, a las cuales se les realizaron las correspondientes lecturas teniendo en cuenta las variables antes mencionadas. 
Se tomaron como criterios de inclusión las láminas diagnosticadas como cervicitis folicular y aquellas con los diagnósticos diferenciales. Fueron criterios de exclusión las láminas diagnosticadas como cervicitis folicular y que al tiempo presentaban infecciones, las de cervicitis folicular con lesiones intraepiteliales de bajo o alto grado, las de mujeres histerectomizadas y aquellas en mal estado (rotas o mal preservadas).

\section{Diseño metodológíco}

Como tipo de estudio para la investigación se utilizó el diseño observacional de tipo descriptivo.

\section{Resultados}

Analizadas las 50 muestras de cervicitis folicular, se encontró en cuanto a la característica celular, que los linfocitos presentaron bordes bien definidos en el $90 \%$, se observaron siempre sueltos, el citoplasma fue escaso y poco

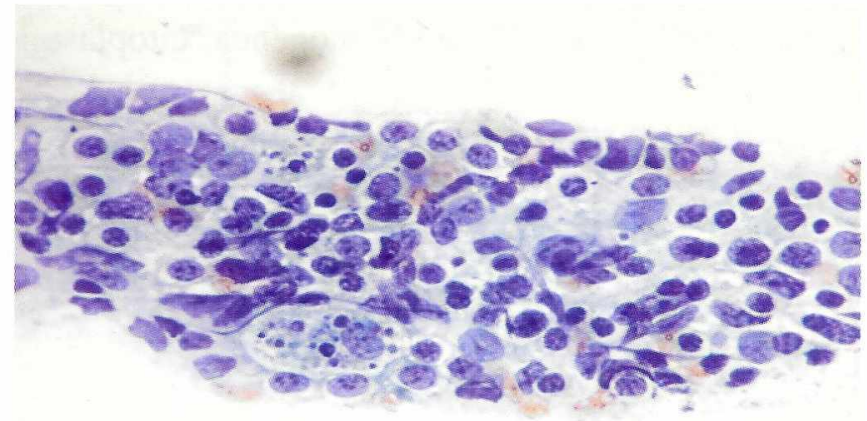

Figura I. Cervicitis folicular: población mixta de linfocitos y dos macrófagos con cuerpos tingibles.

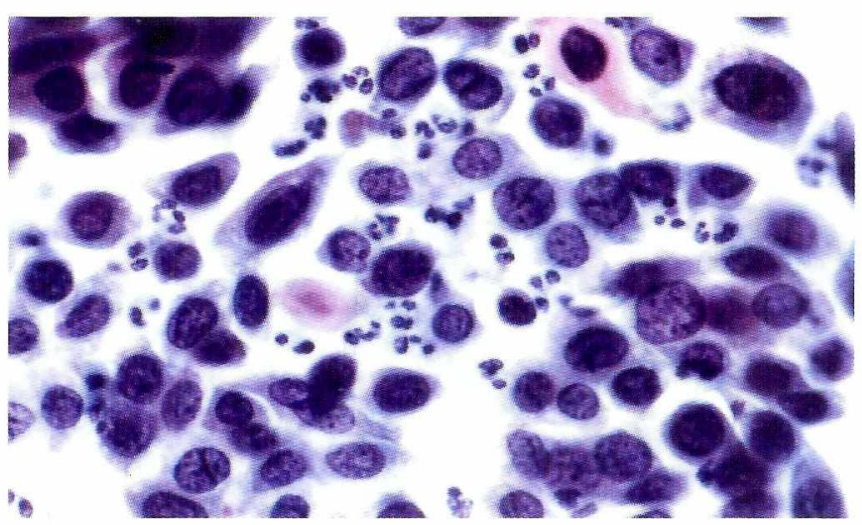

Figura 3. Carcinoma no queratinizante de célula pequeña: los núcleos son hipercromáticos y algunas células están formando grupos. valorable, y el tamaño del núcleo se consideró grande en el $6 \%$, mientras 94\% fueron pequeños (Gráfica 1).

Se presentó en el $70 \%$ cromatina granular, homogénea en el 28\% e hipercromática en $2 \%$ (Gráfica 2). Se observaron nucléolos en el $38 \%$, la membrana nuclear regular en el $90 \%$, mitosis en el $28 \%$ y el fondo de los extendidos fue hemorrágico en $30 \%$, inflamatorio en $46 \%$, limpio en $16 \%$ y sucio en $8 \%$ (Gráfica 3).

De los elementos celulares no epiteliales, se encontró $12 \%$ de plasmocitos, $20 \%$ de histiocitos y $68 \%$ de macrógafos con cuerpos tingibles (Gráfica 4). Los extendidos fueron atróficos en un 52\% (Gráfica 5).

Las 24 muestras correspondientes a los diagnósticos mencionados se diferenciaron de la cervicitis folicular por la característica celular con bordes mal definidos, la descamación celular en conglomerados y sincitios, el citoplasma moderado y vacuolado, el núcleo pequeño, redondo y oval y el fondo, que en las lesiones malignas presentó

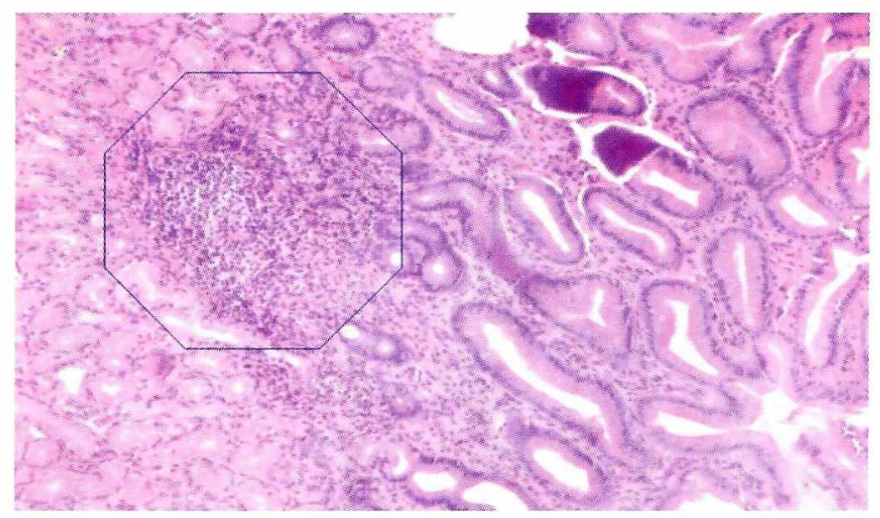

Figura 2. Folículo linfoide sin centro germinativo.

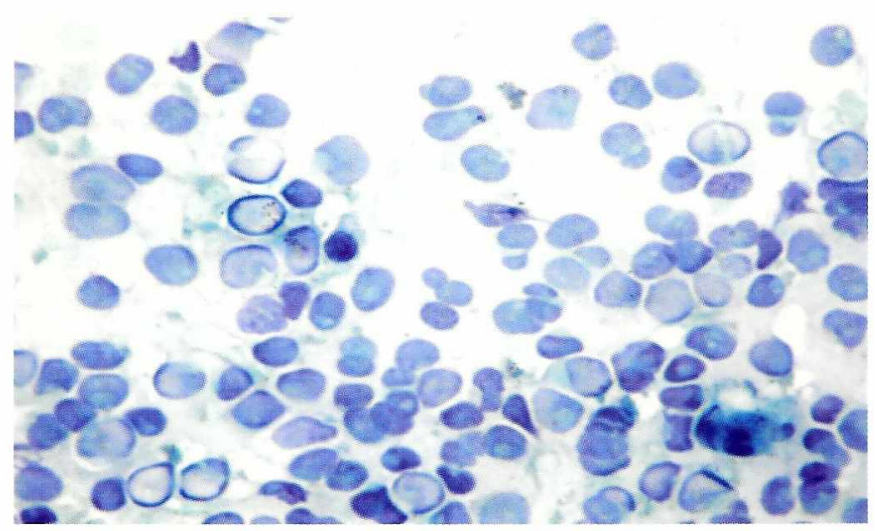

Figura 4. Leucemia: extendido homogéneo con blastos clivados. 
diátesis tumoral y en las reactivas limpio o inflamatorio (Figuras 4 y 5 ).

Terminada la investigación se pudo concluir que la presencia de macrófagos con cuerpos tingibles observada en el 68\% de los casos, obliga a pensar en el diagnóstico de cervicitis folicular.

\section{Discusión}

Ha sido tradicional en la enseñanza y la práctica diaria describir los linfocitos de la cervicitis folicular con cromatina homogénea. Gracias a esta investigación se establece una diferencia, ya que el porcentaje en la cromatina granular es del $70 \%$ comparado con el de homogénea que fue solo del $28 \%$.

Otro aspecto importante es la presencia de linfocitos con depresión central, los cuales en la enseñanza se tomaban como referencia para dar un resultado de cervicitis folicular. En esta investigación se pudo establecer que no es un patrón específico porque no siempre se presentan y en la literatura se conoce poco de ellos.

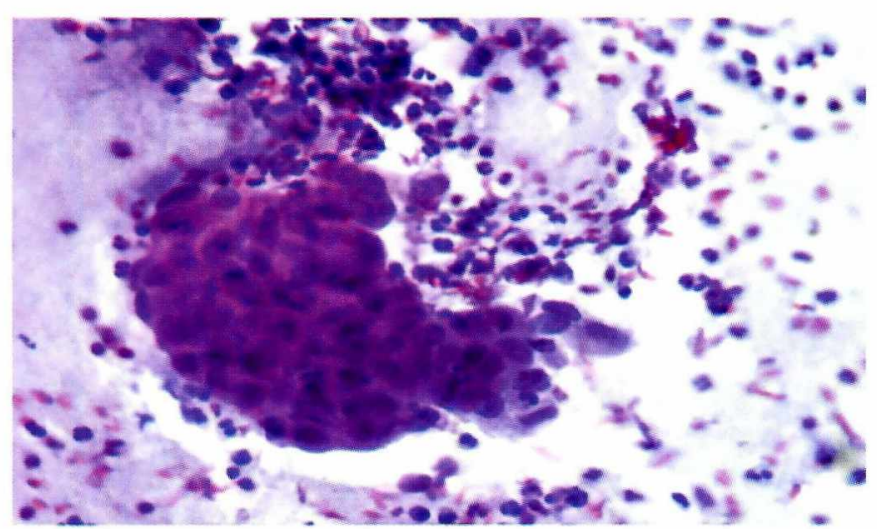

Figura 5.Adenocarcinoma de endometrio:grupo celular cohesivo.

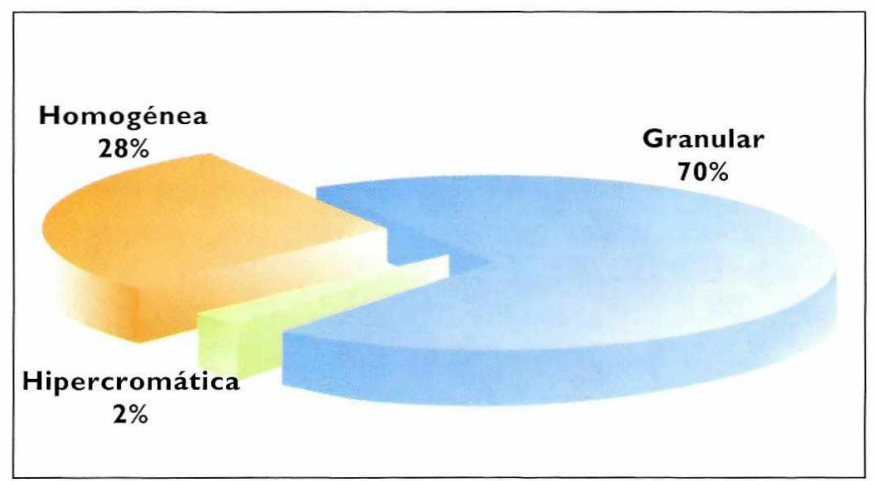

Gráfico 2. Cromatina de los linfocitos.

\section{Conclusiones}

De las 50 láminas diagnosticadas como cervicitis folicular, se observó que el porcentaje de mujeres con extendidos atróficos y no atróficos fue en esencia igual (Gráfica 5).

Algunas láminas con cervicitis folicular mostraron alta concentración de células linfoides, posiblemente debido a la respuesta inmunitaria temprana del folículo linfoide, así como mayor celularidad mixta con histiocitos, macrófagos con cuerpos tingibles y escasos plasmocitos. De acuerdo con la literatura, esto es probable que se deba a la respuesta inmunitaria tardía del folículo linfoide cuando ha desarrollado el centro germinativo.

Debido a la madurez de la respuesta inmunitaria del folículo linfoide, se observó que en una respuesta temprana los núcleos y las mitosis no son evidentes, mientas que en la respuesta tardía si lo son. Dentro de los cambios morfológicos observados en células escamosas,se pudo apreciar aumento del tamaño nuclear, con membrana nuclear regular, cromatina homogénea, citoplasma

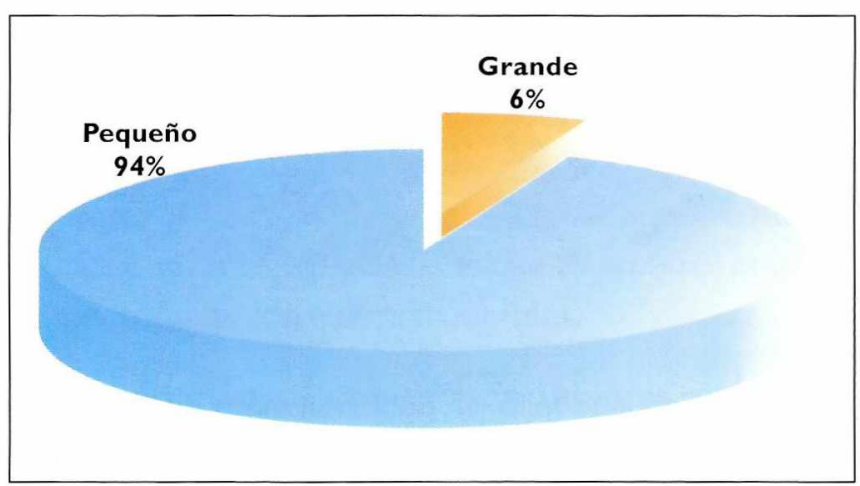

Gráfico I. Núcleos de los linfocitos.

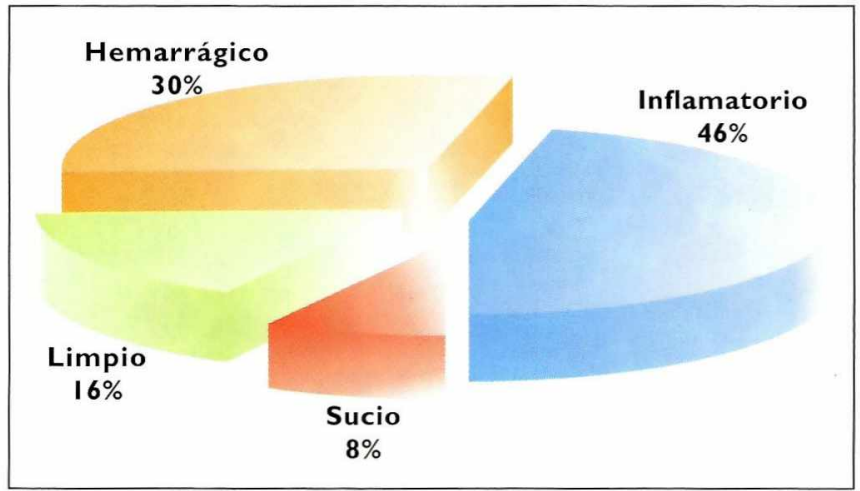

Gráfico 3. Fondo en cervicitis folicular. 


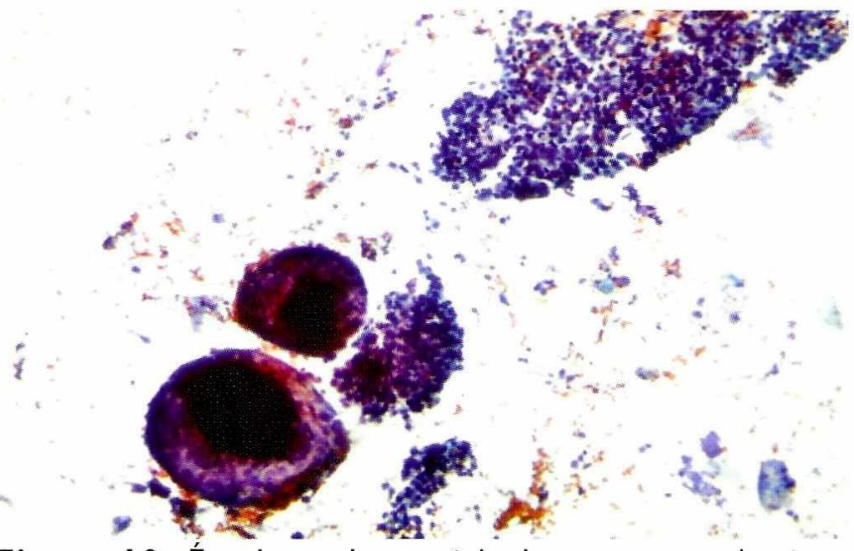

Figura 10. Éxodo endometrial: dos grupos cohesivos inferiores y en la parte superior células poco cohesivas.

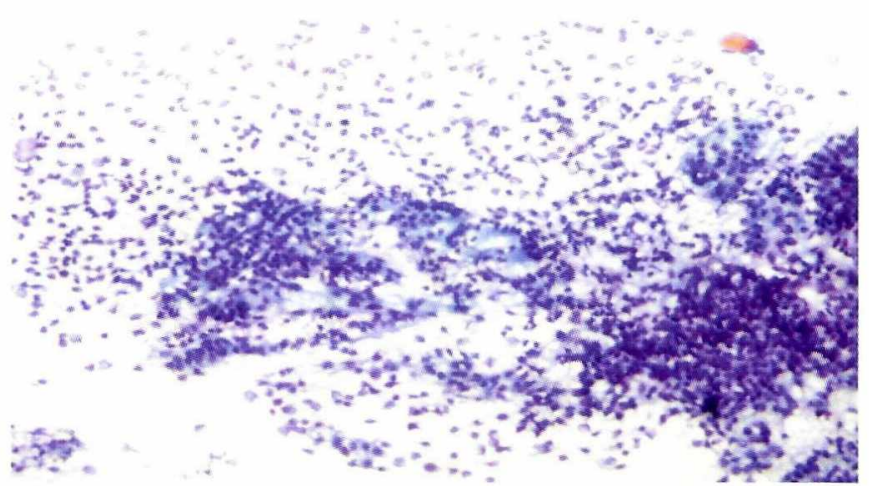

Figura II. Células endocervicales degeneradas: en la parte superior de la foto hay células parecidas a los linfocitos con depresión central, presentes en algunos casos de cervicitis folicular.

\section{Lecturas recomendadas}

- Alonso P, Lazcano E, Hernández M. Cáncer cervicouterino diagnóstico, prevención y control. México: Médica Panamericana, 2000. p.50.

- Atkinson Silverman J. Atlas de dificultades diagnósticas en Citopatología. Madrid: Harcourt; 2000. p. 47.

- Casas A, Salve M. Laboratorio clínico hematología. Madrid Mc Graw Hill Interamericana; 1994. p. 114-25.

- Cibas E, Ducatman B. Diagnastic principles and clinical carrelates. 2 ed. Taranta: Saunders; 2003. p. 19-54.

- García R. Laboratorio y Atlas de Citología. Madrid: Mc Graw Hill Interamericana 1995. p. 63.

- Geneser F. Histología Geneser. Buenos Aires: Médica Panamericana; 1988. p. 350-51.

- FernándezA. Citopatología ginecológica y mamaria. 23 ed. Barcelona: Ediciones Científicas y Técnicas; 1993. p. 350.

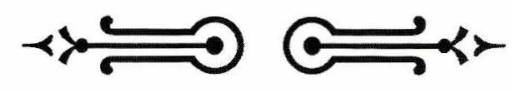

- Kocjan G. Atlas of diagnostic cytopathology. 2 ed. New York. 1997.p.38.

- Ramzy 1. Clinical cytophatology and aspiration biopsy. 2 ed. Texas: Mc Graw Hill Interamericana; 2001. p. 55.

- Roberts T, Alan B. Chronic Iymphocytic cervicitis: Cytologic and histopathologic. Manifestations. Barcelona: International Academy of Cytology; 1975; 19 No 3. p. 235-242.

- Scheneider M, Staemmler H. Atlas de citología diferencial en ginecología. Barcelona: Salvat; 1978.p.61-3.

- Takahashi M. Atlas color citología del cáncer 23 ed Buenos Aires: Médica Panamericana; 1985. p. 182,518,25.

- Turgen H, Beur S. Diagnóstico Citológico en Ginecología. Barcelona: Toray, 1983. p.157-8.

- Wham A, Dydney T. Tratado de histología. 4 ed. Barcelona: Interamericana; 1965. p. 370-75. 